\title{
Radiologic features of a pyrophosphate-like arthropathy associated with long-term dialysis
}

\author{
Ethan M. Braunstein, M.D. ${ }^{1}$, Kathleen Menerey, M.D. ${ }^{2}$, William Martel, M.D. ${ }^{1}$, \\ Richard Swartz, M.D. ${ }^{3}$, and Irving H. Fox, M.D. ${ }^{2}$ \\ Departments of ${ }^{1}$ Radiology, ${ }^{2}$ Internal Medicine, Rackham Arthritis Research Unit, \\ and ${ }^{3}$ Department of Internal Medicine, Division of Nephrology, University of Michigan, Ann Arbor, Michigan, USA
}

\begin{abstract}
In a series of 28 long-term dialysis patients with musculoskeletal complaints, the radiologic findings in six cases resembled those occurring in the arthropathy of idiopathic calcium pyrophosphate dihydrate deposition (CPPD) disease. These findings included osteophytes, subchondral cysts, and cartilage loss in the metacarpophalangeal joints, patellofemoral joints, wrists, and shoulders. Chondrocalcinosis was present in three of the six cases. There were no significant differences in renal function or levels of serum calcium, phosphorus, iron, ferritin, aluminum, or parathormone between these patients and a control group matched for sex and age. Long-term dialysis may be associated with a metabolic arthritis similar to the arthritis which occurs in CPPD deposition disease. The etiology may include deposition of CPPD crystals, hydroxyapatite, or other calciumcontaining substances in joints, or it may be related to a number of dialysis-induced metabolic abnormalities.
\end{abstract}

As dialysis enables more patients with chronic renal failure to survive longer, the effects both of the primary disease and the dialysis process are becoming manifest. Musculoskeletal changes caused by renal disease and consequent secondary hyperparathyroidism are well-known, but arthropathy associated with dialysis itself has only recently been recognized. There have been several reports in the radiologic literature of erosive intra-articular changes both in the small joints of the hand [12]

Address reprint requests to: Ethan M. Braunstein, M.D., Department of Radiology, Indiana University Hospital, $926 \mathrm{~W}$. Michigan St., Indianapolis, IN 46223, USA and shoulder [2]. Whether these changes represent arthritis with secondary bone erosion or bone erosion secondary to hyperparathyroidism is controversial [15].

Descriptions of a non-erosive arthritis of the hands similar to idiopathic calcium pyrophosphate dihydrate deposition (CPPD) disease are less frequent [3]. We prospectively evaluated a series of patients on hemodialysis further to study this arthropathy. As idiopathic CPPD deposition disease resembles the arthropathy of hemochromatosis and may indeed represent the same pathophysiologic process, we also attempted to determine if the radiologic findings were caused by the secondary hemochromatosis of iron overload in this population.

\section{Material and methods}

We prospectively studied a series of 28 patients in our outpatient dialysis program. All patients had musculoskeletal complaints. The study included radiographic joint surveys of the hands, wrists, shoulders, knees, and hips. Radiographs of any other symptomatic areas of the musculoskeletal system were also obtained. Patients having radiographic evidence of an arthropathy similar to that of idiopathic CPPD deposition disease were separated and matched according to age and sex with other members of the group who did not have evidence of arthropathy. Patients were compared according to clinical and biochemical criteria to determine whether there was any correlation between these criteria and the presence or absence of arthropathy.

Data included age, sex, number of years on dialysis, volume of blood received during transfusion, use of iron therapy, and presence or absence of clinical signs and symptoms of liver disease or hemochromatosis. Biochemical tests on blood included blood urea nitrogen (BUN), serum creatinine, calcium, phosphorus, iron, ferritin, parathormone, aluminium, and total iron binding capacity.

One of the coauthors (R.S.) then evaluated the patient and control groups more extensively for hyperparathyroidism. Patients were classified as definitely having hyperparathyroidism if they had elevated serum alkaline phosphatase and para- 
Table 1. Number of patients with biochemical abnormalities of blood

\begin{tabular}{lll}
\hline Blood parameter & $\begin{array}{l}\text { Arthritis group } \\
\text { (Total } n=6)\end{array}$ & $\begin{array}{l}\text { Control group } \\
\text { (Total } n=6)\end{array}$ \\
\hline BUN & 6 & 6 \\
Creatinine & 6 & 6 \\
Calcium & 1 & 1 \\
Phosphorus & 5 & 5 \\
Iron & 1 & 0 \\
TIBC & 0 & 2 \\
Ferritin & 4 & 6 \\
Parathormone & 5 & 4 \\
Aluminum & 4 & 4 \\
\hline
\end{tabular}

$\mathrm{TIBC}=$ Total iron binding capacity

Table 2. Number of arthritis patients with radiographic abnormalities at various sites (Total $n=6$ )

\begin{tabular}{ll}
\hline Metacarpophalangeal & 6 \\
Wrists & 4 \\
Shoulders & 1 \\
Knees & 2 \\
Hips & 1 \\
Spine & 1 \\
\hline
\end{tabular}

thormone, radiographic evidence of subperiosteal resorption, histologic evidence of parathyroid hyperplasia, and favorable response to parathyroidectomy. Patients were classified as possibly having hyperparathyroidism if they had elevated serum alkaline phosphatase and parathormone, few signs and symptoms, and equivocal subperiosteal resorption on radiographs. Hyperparathyroidism was considered doubtful if alkaline phosphatase was normal, parathormone was slightly elevated, and radiographs showed no subperiosteal resorption.

The patient data were stored and analyzed by the University of Michigan Clinical Research Center CLINFO data base management system on a VAX 11/730 minicomputer.

\section{Results}

Six of 28 patients showed radiographic evidence of metabolic arthritis, and 22 had no evidence of such arthritis. Of these 22, 6 were matched with the patients with positive radiographs according to age, sex, and length of dialysis.

In the arthritis group, there were four men and two women. The age range was 48 to 70 years, with a mean of 60 years. In the control group, there were four men and two women. The age range was 49 to 65 years, with a mean of 60 years. Patients with arthritis had been on hemodialysis,

Fig. 1. Seventy-year-old woman on chronic dialysis. Notice the arthropathy typical of CPPD disease, including cartilage loss at the metacarpophalangeal joints and hook-like osteophytes of the metacarpal heads. There also is cartilage loss at the radiocarpal joint and some bone fragmentation
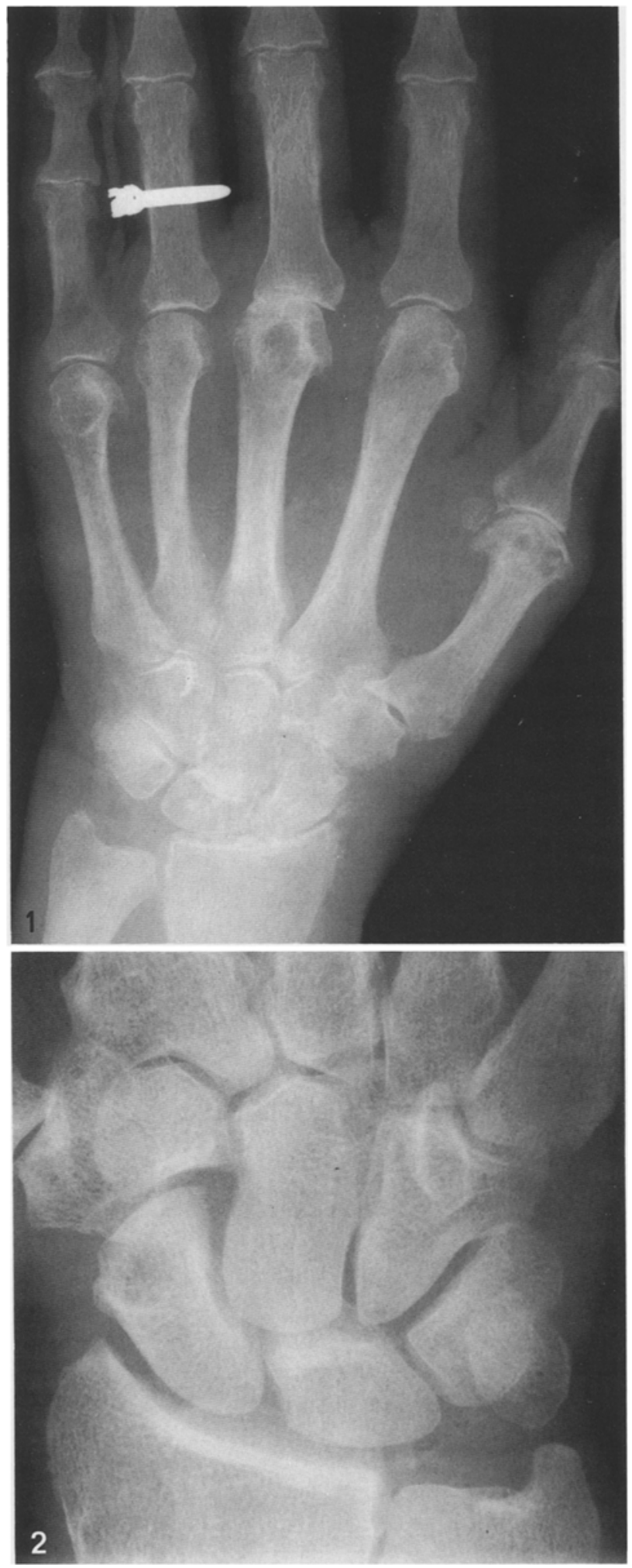

Fig. 2. Chondrocalcinosis of the triangular fibrocartilage in a 60 -year-old man on dialysis for 5 years. He had no clinical or laboratory evidence of hemochromatosis, and he had received no blood transfusions. The patient had hand and wrist findings similar to those of idiopathic CPPD disease 
chronic peritoneal dialysis, or a combination of the two for 2 to 13 years, with a mean of 4.7 years. Control patients had been on dialysis from 1 to 14 years, with a mean of 7 years. Only two arthritis patients had received transfusions (1 and 8 units). All control patients received transfusions of comparable volume. Two arthritis patients and four control patients had received supplemental iron therapy. On physical examination, no patient in either group had signs of liver failure.

The numbers of patients with various biochemical abnormalities in each group are summarized in Table 1. BUN ranged from $52-107 \mathrm{mg} / 100 \mathrm{ml}$ in the arthritis group and from $35-144 \mathrm{mg} / 100 \mathrm{ml}$ in the control group. Creatinine ranged from $10-18 \mathrm{mg} / 100 \mathrm{ml}$ in the arthritis group, and from $11-22 \mathrm{mg} / 100 \mathrm{ml}$ in the control group. One patient in each group had mild hypocalcemia. Five patients in each group had elevated phosphorus levels, with a maximum of $16 \mathrm{mg} \%$ in the arthritis group and $10 \mathrm{mg} \%$ in the control group. Five patients in each group had elevated levels of serum parathormone. Patients in the arthritis group had a mean of 4.5 of these biochemical abnormalities per patient; patients in the control group had a mean of 4.7 abnormalities per patient. Thus, there was no significant difference in the total number of abnormalities per patient in the two groups.

According to the clinical and biochemical criteria in Materials and Methods one patient in the arthritis group was definitely hyperparathyroid, three patients were possibly hyperparathyroid, and two patients were probably not hyperparathyroid. In the control group, one patient was definitely hyperparathyroid, and five patients were probably hyperparathyroid. None had radiographic evidence of arthritis.

Radiographic findings in the arthritis group are summarized in Table 2. Radiographs in the control group showed no subperiosteal resorption or arthritis. In the arthritis group, the pattern of radiographic change was identical to that of idiopathic CPPD deposition arthropathy. That is, there were subchondral cyst-like lucencies, osteophyte formation, and subchondral sclerosis at metacarpophalangeal joints in all cases, and radiocarpal involvement in four of six patients (Fig. 1). There was chondrocalcinosis in three cases. In one patient (Fig. 2), there was unilateral calcification of the triangular fibrocartilage of the wrist. There was articular cartilage calcification in the shoulder of one patient. In one symphysis pubis, there was fibrocartilage calcification of the disk. No patient had chondrocalcinosis of more than one joint. Unfortunately, none of the patients consented to joint aspiration; whether or not crystals were present is unknown.

\section{Discussion}

Prolonged dialysis may be associated with progressive deterioration of both structure and function of joints, with or without acute episodes of synovitis [4]. Many authors have observed radiologic evidence of erosive arthritis associated with dialysis. Sundaram et al. [15] noted articular and periarticular erosions in up to $30 \%$ of patients on maintenance hemodialysis. These erosions seemed to be associated with secondary hyperparathyroidism, but they were not correlated with subperiosteal resorption of extra-articular sites. Moreover, patients with these findings had no arthralgias or arthritis. Other authors $[5,12]$ have also attributed these erosions to hyperparathyroidism rather than to arthritis of another etiology, although the typical distribution was in the small joints of the hands. Erosions in large joints, such as the shoulders, have also been reported [2].

In our series, the arthropathy was not characterized by juxtachondral marginal erosions, but rather resembled the degenerative pattern seen in idiopathic CPPD deposition disease. That is, it occurred in metacarpophalangeal joints, and it was associated with joint collapse and subchondral pseudocysts in the hand and wrist (Fig. 3) [8]. Other joints more typically affected in idiopathic degenerative joint disease were also involved (Fig. 3). In one case, degenerative change was so extensive that the appearance of the spine resembled neuropathic arthropathy. This is interesting in view of a report [7] on 10 dialysis patients in whom a destructive spinal arthropathy, resembling a neuropathic spine or advanced CPPD deposition disease, was noted. In the illustrated cases, CPPD crystals were not found in surgical specimens taken at spinal laminectomy and fusion, but one patient did have hydroxyapatite crystals. In our series, one patient did have severe degenerative spinal changes resembling those of a neuropathic spine (Fig. 4), but these changes were not in the cervical spine as had previously been described in the literature [7].

Three of our six patients had chondrocalcinosis (Table 2). An erosive arthritis in primary and secondary hyperparathyroidism has been described in association with chondrocalcinosis [11], but no patients in that series had undergone dialysis. Moreover in our series four of six patients with arthritis were probably or definitely hyperparathyroid, and all six controls were probably or definitely hyper- 

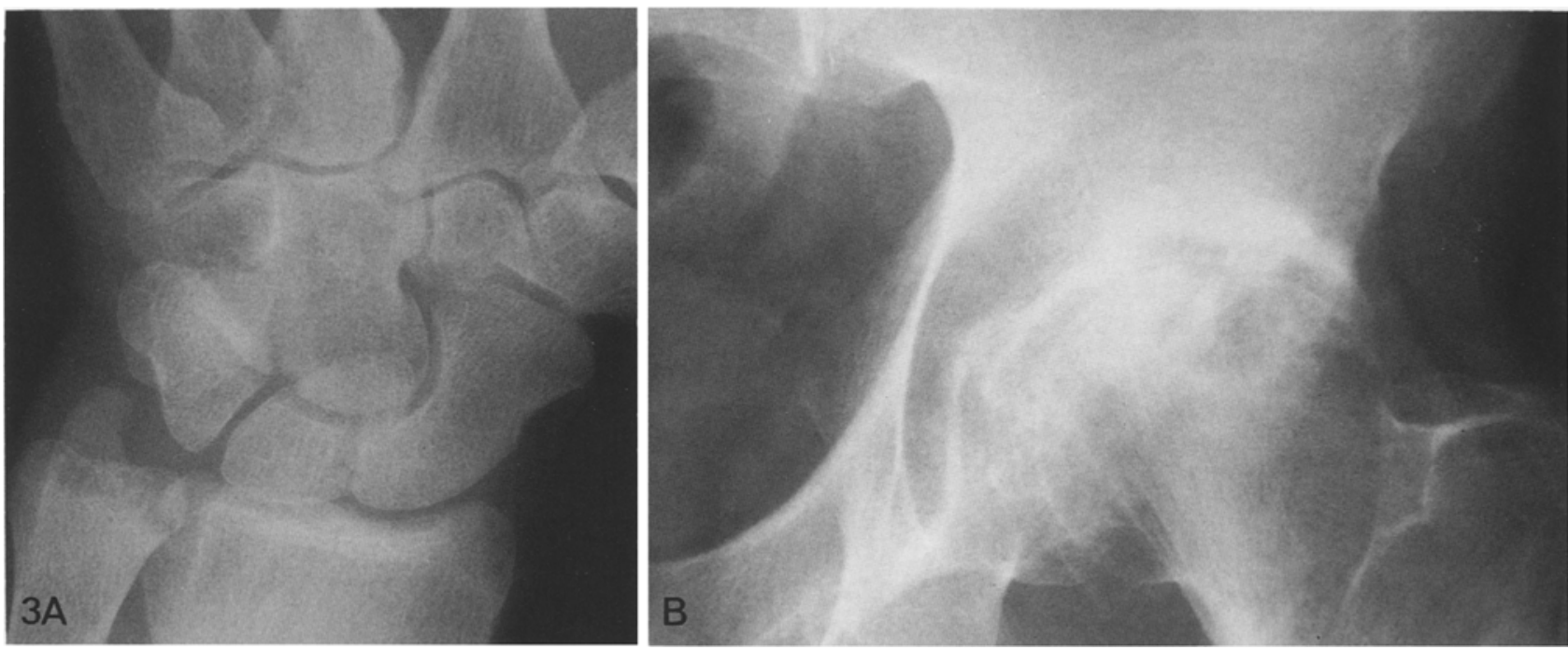

Fig. 3. A Multiple, coalescent, subchondral, cyst-like lucencies of the distal ulna in a 61-year-old man on dialysis. The coalescence of these small cysts is typical of CPPD disease. B Degenerative joint disease of the left hip, characterized by cartilage loss, osteophytes, and subchondral cyst-like lucencies of the femoral head in a 58-year-old man on chronic dialysis. Although these findings are characteristic of degenerative joint disease, this patient also had changes in the hands and wrists similar to those of CPPD disease

parathyroid. Therefore, the arthropathy could not be shown to be exclusively related to hyperparathyroidism.

Although it may still be argued that the arthropathy in our patients may be caused by hyperparathyroidism and that the patients in our control group may ultimately develop such an arthropathy, there were two patients in the arthritis group who probably or definitely were not hyperparathyroid, and there were no such patients in the control group. The patients who were not hyperparathyroid (Fig. 2) had findings similar to those in the arthritis group. Of course, we also cannot exclude chance association of idiopathic pyrophosphate deposition disease in our arthritis group; nevertheless, the presence of six such patients in a small series of 28 suggests a correlation.

Ellmann et al. [3] described two cases of CPPD deposition disease in patients with chronic renal failure. In one case, there was diffuse chondrocalcinosis and, in the other, CPPD crystals were aspirated from the elbow. Although the radiographic pattern in our patients is the same as Ellman describes, none of our patients had erosions. Since none of the patients consented to joint aspiration, we do not know if CPPD crystals were present.

The arthropathy of hemochromatosis may be identical [8] or similar [1] to that of CPPD deposition disease. Indeed, recent work [14] has shown that calcium pyrophosphate crystals are found in association with cartilage degeneration in hemochromatosis even when iron is not present in the chondrocytes. Whether such crystals are the cause of the degenerative change or simply an associated phenomenon is still unclear. One autopsy series [10] showed that chronic iron administration had resulted in tissue iron deposition. In another study [13] subclinical organ dysfunction resulted from transfusion iron overload and resembled hemochromatosis. Portal fibrosis was demonstrated in these patients. The pathogenesis of the arthritis may result from inhibition of pyrophosphatase by iron, with resultant deposition of intra-articular CPPD crystals. Iron itself has been found in the synovial lining in patients with hemochromatosis [6], but it does not necessarily cause the arthropathy.

In this context, only one of our arthritis patients and none of the controls had elevated serum iron. None of the arthritis patients had an elevated total iron-binding capacity and, although four of six arthritis patients had elevated serum ferritin, all six controls also had elevated serum ferritin. Indeed, there were no differences between the arthritis group and the control group in any of the criteria studied, particularly in severity of the renal disease, number of years on dialysis, serum calcium, parathormone, or any other clinical or laboratory findings.

In another study [9], dialysis patients with clinical, rather than radiologic, evidence of arthritis also had no differences from controls with regard to several clinical and laboratory criteria, including ferritin, parathormone, calcium, phosphate, HLA 


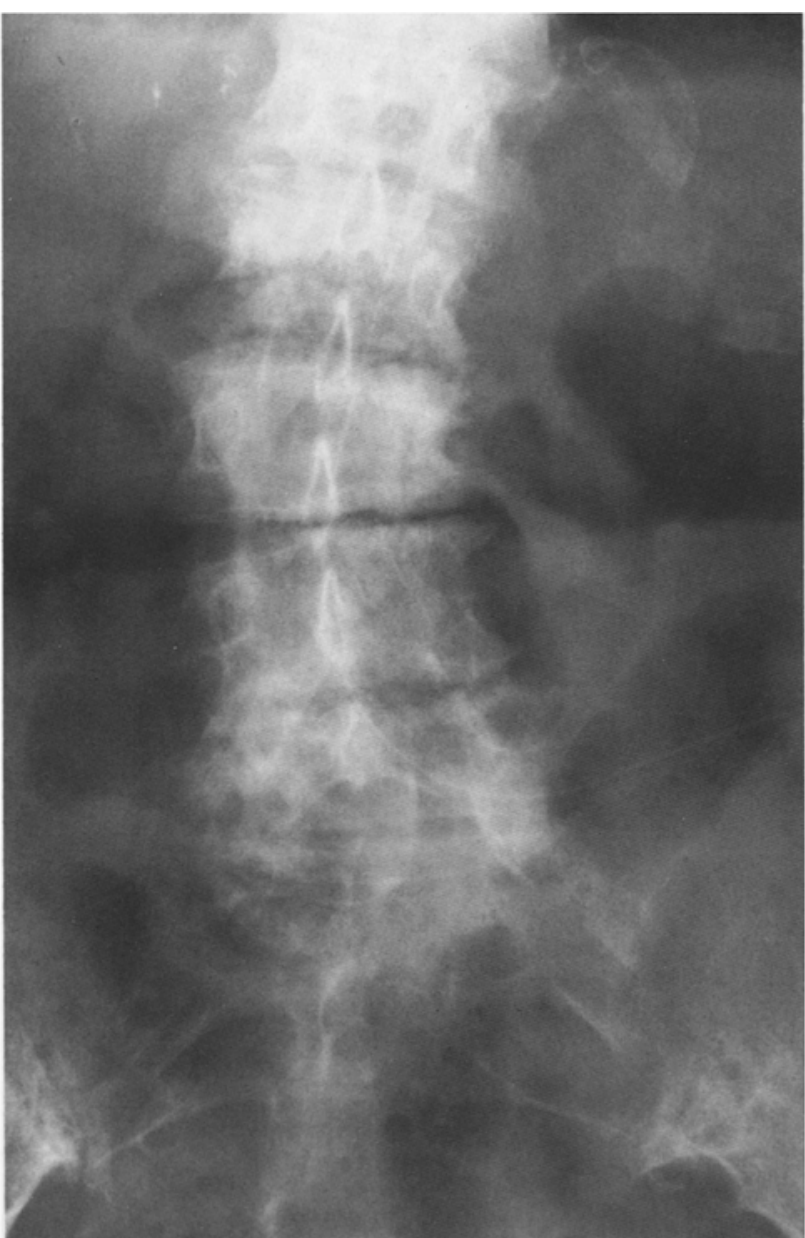

Fig. 4. Severe degenerative changes in the lumbar spine in the same case as Fig. 1. The findings are similar to severe degenerative changes characteristic of patients with advanced CPPD disease or neuropathic spine. Also notice the cystic and sclerotic changes of the sacroiliac joints

halotyping for iron overload arthropathy, or aluminum. However, the patients with clinical arthritis had more abnormal findings together than the patients without arthropathy. In our study, there was no difference in the number of abnormalities per patient. However, it is still possible that these metabolic abnormalities may cause an arthropathy, even if the arthropathy is not clinically or radiographically present in all patients.

In summary, we have found that $21 \%$ of 28 chronic dialysis patients with musculoskeletal complaints had an arthropathy similar to that of idiopathic CPPD deposition disease. Although the number of cases is small and we cannot exclude the possibility that, out of 28 patients on dialysis, there were six with idiopathic CPPD deposition disease, we feel that this is most unlikely in the context of the number of metabolic abnormalities involved in chronic renal failure and dialysis. We cannot explain the pathogenesis of this arthropathy on the basis of the clinical and laboratory data available. Perhaps calcium pyrophosphate or some other abnormal dialysis metabolite is deposited in cartilage or synovium, or the renal failure or the dialysis itself leads to a primary abnormality in the cartilage matrix with secondary crystal deposition. Whatever the pathogenesis, a degenerative arthropathy should be included among the complications of long-term dialysis.

Acknowledgements. We thank Mr. S. Schmaltz for assistance in data storage and analysis, and Ms. K. Jarvenpaa and the University of Michigan Clinical Research Center nurses for patient management. This work was partially supported by the University of Michigan Clinical Research Center through a grant from the Division of Research Resources (5MO1 RR42), National Institutes of Health

\section{References}

1. Adamson TC III, Resnik CS, Guerra J Jr, Vint V, Weisman MH, Resnick D (1983) Hand and wrist arthropathies of hemochromatosis and calcium pyrophosphate deposition disease: Distinct radiographic features. Radiology 147:377

2. Bonavita JA, Dalinka MK (1980) Shoulder erosions in renal osteodystrophy. Skeletal Radiol 5:105

3. Ellman ME, Brown N, Katzenberg CA (1979) Acute pseudogout in chronic renal failure. Arch Intern Med 139:795

4. Goldstein S, Winston E, Chung TJ, Chopra S, Pariser K (1985) Chronic arthropathy in long-term hemodialysis. Am J Med 78:82

5. Hamilton S, Knickerbocker WJ (1982) Periarticular erosions in the hands and wrists in hemodialysis patients. Clin Radiol 33:19

6. Hirsch JH, Killien C, Troupin RH (1976) The arthropathy of hemochromatosis. Radiology 118:591

7. Kuntz D, Naveau B, Bardin T, Drueke T, Treves R, Dryll A (1984) Destructive spondyloarthropathy in hemodialyzed patients. Arthritis Rheum 27:369

8. Martel W, McCarter DK, Solsky M, Good AE, Hart WR, Braunstein EM, Brady T (1981) Further observations on the arthropathy of calcium pyrophosphate crystal deposition disease. Radiology $141: 1$

9. Menerey K, Swartz R, Brown M, Braunstein E, Brown C, Fox I (1987) Metabolic basis of arthropathy in dialysis patients (personal communication)

10. Pitts TO, Barbour GL (1978) Hemosiderosis secondary to chronic parenteral iron therapy in maintenance hemodialysis patients. Nephron 22:316

11. Resnick DL (1974) Erosive arthritis of the hand and wrist in hyperparathyroidism. Radiology 110:263

12. Rubin LA, Fam AG, Rubenstein J, Campbell J, Saiphoo C (1984) Erosive azotemic osteoarthropathy. Arthritis Rheum 27:1086

13. Schafer AI, Cheron RG, Dluhy R, Cooper B, Gleason RE, Soeldner JS, Bunn HF (1981) Clinical consequences of acquired transfusional overload in adults. $N$ Engl $J$ Med $304: 319$

14. Schumacher HR (1982) Articular cartilage in the degenerative arthropathy of hemochromatosis. Arthritis Rheum $25: 1460$

15. Sundaram M, Wolverson MK, Heiberg E, Grider RD (1981) Erosive azotemic osteodystrophy. AJR 136:363 